\title{
ARTICLE
}

Behaviour, Psychology and Sociology

\section{Do bedroom screens and the mealtime environment shape different trajectories of child overweight and obesity? Research using the Growing Up in Scotland study}

\author{
Alison Parkes ${ }^{1} \cdot$ Michael Green ${ }^{1} \cdot$ Anna Pearce $^{1}$ \\ Received: 21 January 2019 / Revised: 6 November 2019 / Accepted: 17 November 2019 / Published online: 11 December 2019 \\ (c) The Author(s), under exclusive licence to Springer Nature Limited 2019
}

\begin{abstract}
Objective To investigate how mealtime setting, mealtime interaction and bedroom screens are associated with different trajectories of child overweight and obesity, using a population sample.

Methods Growth mixture modelling used data from children in the Growing Up in Scotland Study born in 2004/5 (boys $n=2085$, girls $n=1991$ ) to identify trajectories of overweight or obesity across four time points, from 46 to 122 months. Using data from children present at all sweeps, and combining sexes $(n=2810)$, mutually adjusted associations between primary exposures (mealtime setting, mealtime interaction and bedroom screens) and trajectory class were explored in multinomial models; controlling for early life factors, household organisation and routines, and children's diet patterns, overall screen use, physical activity and sleep.

Results Five trajectories were identified in both sexes: Low Risk (68\% of sample), Decreasing Overweight (9\%), Increasing Overweight (12\%), High/Stable Overweight (6\%) and High/Increasing Obesity (5\%). Compared with the Low Risk trajectory, High/Increasing Obesity and High/Stable Overweight trajectories were characterised by early increases in bedroom screen access (respective relative risk ratios (RRR) and 95\% confidence intervals: 2.55 [1.30-5.00]; 1.62 [1.01-2.57]). An informal meal setting (involving mealtime screen use, not eating in a dining area and not sitting at a table) characterised the High/Increasing Obesity and Increasing Overweight trajectories (respective RRRs compared with Low Risk trajectory: 3.67 [1.99-6.77]; 1.75 [1.17-2.62]). Positive mealtime interaction was associated with membership of the Increasing Overweight trajectory (RRR 1.64 [1.13-2.36]).

Conclusion Bedroom screen access and informal mealtime environments were associated with higher-risk overweight and obesity trajectories in a representative sample of Scottish children, after adjusting for a wide range of confounders. Findings may challenge the notion that positive mealtime interaction is protective. Promoting mealtimes in a screen-free dining area and removing screens from bedrooms may help combat childhood obesity.
\end{abstract}

\section{Introduction}

Ecological theories underline the critical proximal role of the family environment for children's healthy weight gain [1]. This paper focuses on the role of two specific aspects of

Supplementary information The online version of this article (https:// doi.org/10.1038/s41366-019-0502-1) contains supplementary material, which is available to authorised users.

Alison Parkes

Alison.parkes@glasgow.ac.uk

1 MRC/CSO Social and Public Health Sciences Unit, University of Glasgow, Glasgow, UK the family environment: family mealtime environments and screens in children's bedrooms. In many western countries, both these aspects of family life have undergone a societal shift that parallels the development of the obesity epidemic. Time pressures on working parents, increased reliance on convenience foods (relative to healthier options) and the growth of technology have altered the character of family mealtimes, with more families adopting an informal style of eating accompanied by mealtime screen use [2]. Rapid growth in new forms of affordable screen technology, including portable devices such as tablets and mobiles, as well as TV and computers, has facilitated children's ownership and bedroom use [3, 4]. Family mealtimes and bedroom screens are likely to be important influences on children's food consumption and screen use, and both offer 
potentially well-defined, actionable intervention targets. This paper aims to further our understanding of the likely benefits of specific changes to the family mealtime environment and access to bedroom screens for children's weight status.

\section{Family mealtime environment}

The social and physical environments of family meals provide an important context for routines and rituals associated with appetite stimulation and regulation, shaping opportunities for parental modelling and oversight of children's food intake [5, 6]. Empirical evidence for the effects of mealtime social interaction is, however, inconclusive. Positive interaction was associated with lower child BMI in two cross-sectional studies [5, 7], but others have linked fewer mealtime arguments to an indulgent parental feeding style making few demands on the child, and to higher energy intake $[8,9]$. Physical aspects of mealtime environment have been explored most in relation to mealtime television use, linked to child overweight in a recent metaanalysis [10]. Possible mechanisms include exposure to obesogenic food advertising (which can have both immediate effects on dietary intake, and longer term effects on fast food preferences [11, 12]); adverse effects of mealtime screen use on appetite regulation and control [13]; and less parental monitoring of food consumption [14]. The social and physical context of mealtimes may have independent and/or interactive effects on children's diet, although it is not clear whether mealtime screen use compromises or promotes a positive atmosphere $[15,16]$.

\section{Bedroom screen access}

Longitudinal studies indicate that bedroom TV is a risk factor for childhood obesity, via mechanisms that may include reduced sleep or physical activity, and/or increased screen use, food advertising exposure and snacking on junk food $[17,18]$. Other bedroom screens such as computers, while less well studied, may also link to obesity risk via similar mechanisms [19].

Existing studies of the family mealtime environment and access to bedroom screens have generally focused on associations with children's weight status at a single time point, where establishing temporal precedence is difficult and information on background confounding may be limited. We aim to establish a clearer picture of the longer-term effects of the mealtime environment and bedroom screens, by investigating their association with different developmental patterns of overweight and obesity over an extended period of time. Across early to middle childhood, we expect to find groups of children at high or increasing risk of overweight and obesity, in addition to children maintaining a healthy weight [20-24]. We hypothesise that bedroom screens and informal mealtime settings will both be associated with membership of higher-risk overweight and obesity trajectories. Furthermore, we hypothesise independent, additive effects of these two factors, due to potentially different underlying mechanisms. We do not make a hypothesis in relation to mealtime social context, as existing evidence is contradictory; but we explore possible interactive effects between mealtime social and physical context.

\section{Methods}

Data were from the first birth cohort of the Growing Up in Scotland study [25], a nationally representative cohort of families with children born between June 2004 and May 2005. Details of the sampling framework are provided elsewhere [26]. Data collection was subject to medical ethical review by the Scotland 'A' MREC committee. All participants provided written informed consent. Families were first interviewed $(n=5217)$ when children were 10 months old, and followed up at 22, 34, 46, 58, 70, 94 and 122 months.

\section{Measures}

Unless otherwise specified, information was supplied by the child's main carer (usually the mother).

\section{Child overweight and obesity}

BMI (weight $(\mathrm{kg}) /$ height $\left.(\mathrm{m})^{2}\right)$ at four time points $(46,70,94$ and 122 months) was calculated from height and weight measurements obtained by trained researchers. Measures three standard deviations or more from the mean were treated as potentially unreliable, and recoded as missing $(n=20$ at 46 months, $n=24$ at 70 and 94 months, $n=2$ at 122 months). Overweight and obesity were defined using age- and sexstandardised International Obesity Task Force cutoffs [27].

\section{Primary exposures}

\section{Mealtime environment}

Two factors reflecting the social and physical mealtime environment were derived from a factor analysis of all items.

\section{Mealtime interaction}

Mealtime interaction was a factor score of two items, each measured at 58 and 122 months (four items, loadings all 0.6), indicating main carer's agreement with "mealtimes are enjoyable for everyone", and "mealtimes give us time to 
talk", with responses on a four-point scale. Repeated items were moderately stable $(r=0.35,0.40)$.

\section{Mealtime setting}

Mealtime setting was a factor score of three items, two measured at 58 and 122 months, and a third at 122 months only (five items, loadings 0.5-0.7). Repeated items were: whether the main meal was eaten in a "dining" area (=kitchen, dining room, combined living/dining room) or "non-dining" area (=living room, bedroom ${ }^{1}$, other); and mealtime screen use (TV only at 58 months, but at 122 months this included other screens). Repeated items were moderately stable $(r=0.42,0.43)$. At 122 months only, an item asked how often the child sat at a table while eating a main meal. Items concerning mealtime screen use and sitting at the table were on a four-point scale.

Scores for mealtime setting and interaction were divided into tertiles. For mealtime interaction, these were labelled "negative", "intermediate" and "positive" interaction, where "positive" indicates mealtimes being rated as enjoyable for everyone and allowing time to talk. For mealtime setting, tertiles were labelled "formal", "intermediate" or "informal", where "informal" indicates greater mealtime screen use, and less use of a table or dining area.

\section{Bedroom screen trajectory}

Bedroom screen trajectory during the study period was measured using growth mixture modelling (GMM) of screen devices present in the child's bedroom at 46, 58, 94 and 122 months. At 46 and 58 months, items asked about television (yes $=1$, no $=0$ ). At 94 and 122 months, items included computers, games consoles, handheld gaming exposure and mobile phones (any device $=1$, none $=0$ ). Three trajectories were identified (details in online file S1): late increase (36\% of the sample), early increase $(27 \%)$, high stable $(37 \%)$

\section{Covariates}

Covariates included early life factors, early diet patterns and household organisation and routines that were potential confounders because of known associations with obesity and one or more primary exposures. Child behaviours at school-age (overall screen time, physical activity and sleep) formed an additional set of covariates, which may act as

\footnotetext{
1 Eating the main meal in the bedroom was uncommon, specified by only $4(0.4 \%)$ families at 58 months, and 26 families $(1.8 \%)$ at 122 months.
}

potential confounders (by indicating family healthy lifestyle), and/or (for bedroom screens) as potential mediators.

\section{Early life factors}

Child sex, ethnic group, family socio-economic disadvantage and maternal BMI predict children's higher-risk BMI, overweight and/or obesity trajectories [20, 23, 24] and are associated with one or more primary exposures [5, 7, 17, 28]. Mother's ethnicity was coded as White or Minority. Multiple aspects of socio-economic disadvantage included (1) mother aged under 20 at the child's birth; (2) mother's education level (fivefold National Vocational Qualifications classification); (3) large family (three or more children) at 10 months; (4) household poverty score (based on lowest income quintile, receiving income support, neither parent in paid employment, social rented housing, all at 10, 22 and 34 months); and (5) lone parent score (no resident partner at 10, 22 and/or 34 months). Maternal BMI was calculated from height and weight measurements made by trained researchers. Although only available at child age 70 months, we viewed it as an "early life" covariate likely to have tracked from earlier years. Additional early life factors viewed as plausible confounders comprised: child birth order, maternal smoking in pregnancy (yes/no), maternal mental health (a factor score combining the Short Form -12 scale [29] at 10 and 34 months, and the Depression, Anxiety and Stress Scales [30] at 22 months) and infant feeding (two aspects at 10 months: breastfeeding duration, and age at first introduction of solids).

\section{Early diet patterns}

Early diet patterns were considered as a potential confounder, although healthy diets are not consistently associated with children's overweight or obesity [31], while picky eating (where a child has strong food preferences and is reluctant to try new foods) has been related to both overweight and underweight [32]. All primary exposures are associated with a less healthy diet [33-35] and mealtime exposures are associated with diet variety [36]. 'Healthy diet' was based on four items repeated at 22 and 58 months ( 8 in all, standardised alpha $=0.65$ ), concerning the child's consumption of sweets, crisps, fruit and vegetables. Picky diet was based on two items measured at 22 months (standardised alpha $=0.82$ ), concerning how easy it was to feed the child (5-point scale); and whether the child could be described as eating most things, eating a reasonable variety of things, or a fussy eater.

\section{Household organisation and routines}

These potential confounders are associated with children's weight status [23, 37], and with bedroom TV and mealtime 
climate [38, 39]. Home organisation at 58 months used three items from the confusion, hubbub, and order scale, alpha $=0.65$ [40]. Irregular bedtimes were a standardised score based on an item repeated at 58, 70 and 94 months (three items, alpha $=0.70$ ) concerning bedtime regularity on nights before a school day (responses on a 4-point scale: always, often, sometimes and never). Skipping breakfast was a binary measure at 58 months.

\section{Children's behaviours. Screen-time trajectory}

Screen time is associated with an increased risk of obesity [41], and with bedroom screen access and mealtime setting [42, 43]. Daily home screen exposure at 46, 58, 70, 94 and 122 months used items concerning typical weekday and weekend television use (inclusive of video/DVDs). From 58 months, items included the use of computers and games consoles. At 94 and 122 months, items included mobile phones. Scores were divided into three categories: $<2 \mathrm{~h} /$ day, 2 to $<4 \mathrm{~h}$ and $4+$ hours. Screen time trajectory was assigned using GMM (see online file S1). Three trajectories were identified: low (26\% of the sample), medium (55\%) and high (19\%). All showed an increase over time. This was because later measures of screen time were more inclusive (including all screen types) and not due to real increase in TV time. Physical activity: Activity levels are associated with child obesity and bedroom screen access [44, 45]. Activity was measured at 58 and 70 months, using average time spent on moderate to vigorous activities in the past week. Sleep: Shorter sleep duration is associated with obesity and bedroom screens [46, 47]. We used the average typical hours of sleep during a $24 \mathrm{~h}$ day at 70 and 94 months.

\section{Samples used for growth mixture models (GMM) of overweight and obesity, and analysis of trajectory correlates}

GMM of overweight/obesity trajectories used all children with one or more reliable measures of BMI at 46, 58, 70 and 122 months (boys $n=2085$, girls $n=1991$ ). The analysis sample used to explore trajectory correlates was selected from families interviewed at the last time point, 122 months (total $n=3151$ ). We excluded cases not participating in all previous sweeps $(n=338)$, as these lacked longitudinal survey weights; and cases without reliable measures of child BMI at 46, 70, 94 or 122 months $(n=3)$. This gave an analysis sample of 2810 families (boys $n=1432$, girls $n=$ 1378). After applying longitudinal survey weights, representation of sociodemographic characteristics in the analysis sample resembled the baseline sample (respective figures were: ethnic minority mothers $3.3 \%$ vs $4.0 \%$, mothers with no educational qualifications $7.8 \%$ vs $9.6 \%$, lone parent households $19.4 \%$ vs $20.3 \%$, lowest household income quintile $21.9 \%$ vs $21.5 \%$.

\section{Analytic strategy}

GMM identified different trajectories in the probability of being overweight or obese at 46, 70, 94 and 122 months, modelled as an ordered categorical variable (healthy/overweight/obese). Modelling was performed on boys and girls separately using Mplus version 8 [48], allowing for the complex survey design, with missing outcome data handled using Full Information Maximum Likelihood estimation. Various model fit statistics were used to help identify the optimum number of classes, together with considerations of the smallest class size and posterior probabilities of class membership [49]. Smaller Akaike Information Criteria and Bayesian Information Criteria values are preferable, while Entropy values should be close to 1. The Lo, Mendell and Rubin Likelihood Ratio Test (LMR) test indicated whether a model had a better fit than the model with one fewer class.

Class membership was exported into Stata [50] for analysis of trajectory correlates. Missing data in the analysis sample were at low levels (on average $<1.5 \%$ ). Nonetheless, with a large number of potential trajectory correlates to explore, a complete case analysis would have resulted in a loss of $34 \%$ of cases and poorer representation of socioeconomic disadvantage. To guard against potential risk of bias and loss of power, 50 sets of missing data were imputed using multiple chained equations.

Multinomial regression models explored associations between each primary exposure and the overweight/obesity trajectory classification in four stages: (1) unadjusted; (2) adjusted for early life covariates, early diet patterns, household organisation and family routines; (3) further adjusted for additional school-age child behaviours (screen time, physical activity and sleep); and (4) as (3) with mutual adjustment for all primary exposures. We tested for interactions between the two mealtime primary exposures at stage 4. Modelling accounted for complex survey design features and used longitudinal survey weights to adjust for sampling and drop-out.

\section{Results}

\section{Trajectories of overweight and obesity}

For boys and girls, a five-class model was selected (for details see online file $\mathrm{S} 2$ ). In both sexes, the five trajectories followed similar patterns and were named according to the pattern of overweight and obesity, as follows: Low Risk (74\% boys, 65\% girls), Decreasing Overweight (9\% boys, 
Fig. 1 Trajectories of overweight and obesity among boys, $N=2085$. Percentage figures indicate each trajectory's share of the boys' growth mixture model sample. For each trajectory graph, the $x$-axis shows age in months, and the $y$-axis probability of overweight (striped area) or obesity (solid area).

Fig. 2 Trajectories of overweight and obesity among girls, $N=1991$. Percentage figures indicate each trajectory's share of the girls' growth mixture model sample. For each trajectory graph, the $x$-axis shows age in months, and the $y$-axis probability of overweight (striped area) or obesity (solid area).
Low Risk $74 \%$

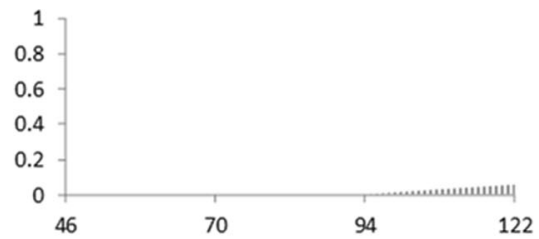

High/Increasing Obesity

$4 \%$

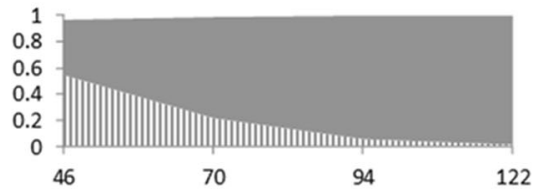

Increasing Overweight

$9 \%$

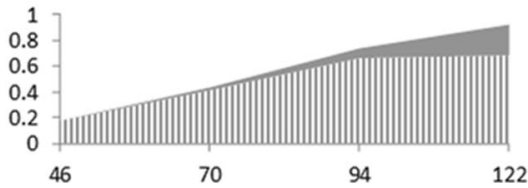

Low Risk 65\%

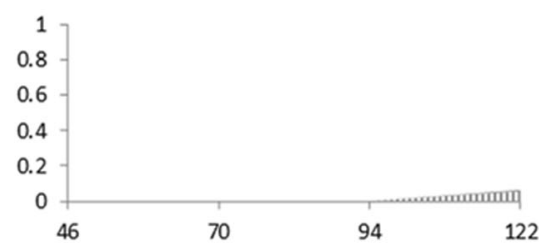

High/Increasing Obesity

$5 \%$

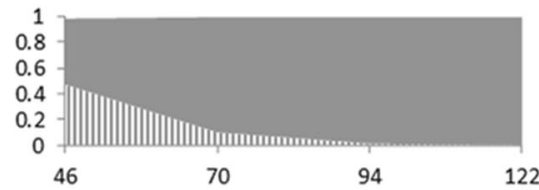

Decreasing Overweight

$9 \%$

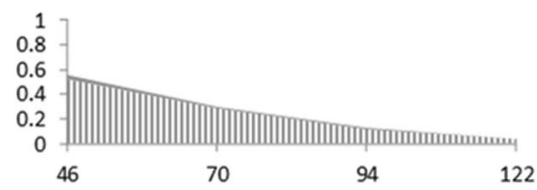

High/Stable Overweight

$4 \%$

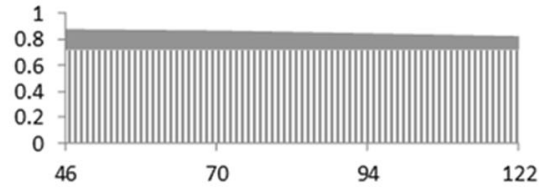

Increasing Overweight

$13 \%$

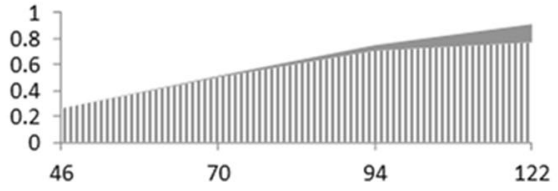

$10 \%$ girls), Increasing Overweight (9\% boys, $13 \%$ girls), High/Stable Overweight (4\% boys, $7 \%$ girls) and High/ Increasing Obesity (4\% boys, $5 \%$ girls). Figures 1 and 2 show the probability of overweight and obesity in each trajectory, for boys and girls.
As boys' and girls' trajectories were similar, analysis of trajectory correlates was performed on the combined sample, checking for sex differences using interaction terms. The distribution of trajectory classes in the analysis sample (68\% Low Risk, 9\% Decreasing Overweight, 12\% 
Table 1 Child BMI z-score and weight status for the analysis sample and by trajectory class.

\begin{tabular}{|c|c|c|c|c|c|c|c|}
\hline & & All $(n=2810)$ & Trajectory class & & & & \\
\hline & & & $\begin{array}{l}\text { High/Increasing } \\
\text { Obesity } \\
(n=112)\end{array}$ & $\begin{array}{l}\text { High/Stable } \\
\text { Overweight } \\
(n=167)\end{array}$ & $\begin{array}{l}\text { Increasing } \\
\text { Overweight } \\
(n=333)\end{array}$ & $\begin{array}{l}\text { Decreasing } \\
\text { Overweight } \\
(n=267)\end{array}$ & $\begin{array}{l}\text { Low Risk } \\
(n=1931)\end{array}$ \\
\hline $\begin{array}{l}\text { Child BMI } z \text { - } \\
\text { score }\end{array}$ & & Mean (SE) & Mean (SE) & Mean (SE) & Mean (SE) & Mean (SE) & Mean (SE) \\
\hline 46 months & & $0.49(0.02)$ & $2.27(0.09)$ & $1.87(0.05)$ & $0.72(0.04)$ & $1.48(0.05)$ & $0.07(0.02)$ \\
\hline 70 months & & $0.39(0.02)$ & $2.70(0.06)$ & $1.80(0.04)$ & $1.04(0.04)$ & $1.01(0.04)$ & $-0.09(0.02)$ \\
\hline 94 months & & $0.42(0.02)$ & $2.83(0.05)$ & $1.76(0.04)$ & $1.54(0.04)$ & $0.79(0.04)$ & $-0.12(0.02)$ \\
\hline 122 months & & $0.51(0.03)$ & $2.81(0.04)$ & $1.72(0.05)$ & $1.83(0.04)$ & $0.59(0.04)$ & $0.00(0.02)$ \\
\hline $\begin{array}{l}\text { Child weight } \\
\text { status }\end{array}$ & & $\%$ & $\%$ & $\%$ & $\%$ & $\%$ & $\%$ \\
\hline 46 months & Overweight & 15.6 & 45.5 & 72.7 & 16.2 & 74 & 0 \\
\hline & Obese & 4.2 & 48.9 & 24.5 & 0 & 3.6 & 0 \\
\hline 70 months & Overweight & 13.8 & 14.3 & 73.5 & 40.5 & 43.4 & 0 \\
\hline & Obese & 6.2 & 85.7 & 26.1 & 3.4 & 1.8 & 0 \\
\hline 94 months & Overweight & 15.4 & 3.4 & 66 & 73.5 & 24 & 0 \\
\hline & Obese & 7.5 & 96.6 & 23.7 & 11.7 & 0.3 & 0 \\
\hline 122 months & Overweight & 19.5 & 0 & 81.3 & 78.8 & 0 & 7.2 \\
\hline & Obese & 7.2 & 100 & 7.1 & 17.5 & 0 & 0 \\
\hline
\end{tabular}

$B M I$ body mass index, $S E$ standard error

Increasing Overweight, 6\% High/Stable Overweight and $5 \%$ High/Increasing Obesity) closely resembled that found for the GMM sample overall. Table 1 shows mean BMI $z$ scores with standard errors, and the percentage overweight or obese at each time point for the complete analysis sample and each trajectory class. Over the study period, the prevalence of overweight (including obesity) increased more than fivefold for children in the Increasing Overweight trajectory, with $17 \%$ obese by age 10 . The prevalence of obesity approximately doubled for children in the High/Increasing Obesity trajectory, so that all were obese by age 10 .

\section{Analysis of trajectory correlates}

Table 2 shows sample characteristics for the whole analysis sample, and the distribution of the three primary exposures and covariates within each trajectory class. For the distribution of covariates according to primary exposures, see online file S3.

\section{Association between mealtime environments, bedroom electronics and trajectories of overweight and obesity}

Multinomial regression models examined associations between each of the primary exposures and overweight/ obesity trajectory class, using the Low Risk trajectory as the reference class. Interaction terms for sex x primary exposure were dropped, as all were non-significant: this provides additional justification for combining boys' and girls' trajectories.

First, we describe separate models for each primary exposure (Table 3, stages 1-3). Table 3 part (a) shows the estimated effects of bedroom screen access. High stable and early increasing bedroom screen access were both strongly associated with membership of the High/Increasing Obesity trajectory, and to a lesser degree with High/Stable Overweight and Increasing Overweight trajectories (stage 1, unadjusted). Adjusting for early life factors, household organisation and routines and diet patterns in stage 2 attenuated these effects, but there were still clear associations between bedroom screen access and the High/Increasing Obesity trajectory (bordering statistical significance for high stable screen access). Stage 3 adjustment for potential mediators (overall screen exposure, sleep and physical activity) produced relatively little change in the magnitude of effect estimates.

Table 3 part (b) shows estimated effects of meal setting. Intermediate and informal settings were associated with the High/Increasing Obesity and Increasing Overweight trajectories, with the effect of informal setting strongest for the former class (stage 1, unadjusted). Stage 2 adjustment attenuated the effect of informal setting on High/Increasing Obesity trajectory membership, but other effects remained similar. Adjusting for school-age child behaviours (stage 3) produced little further change in the estimates. 


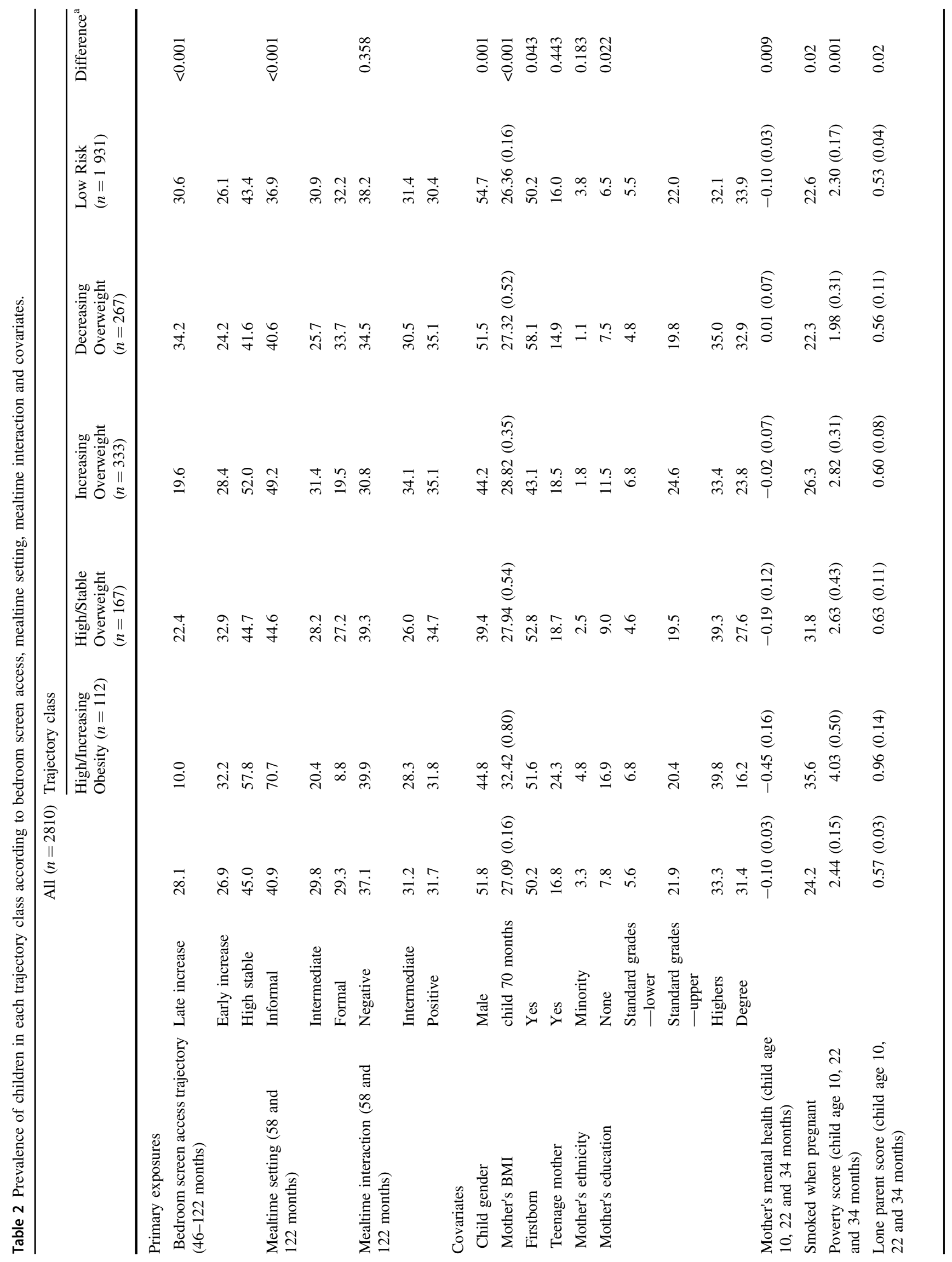




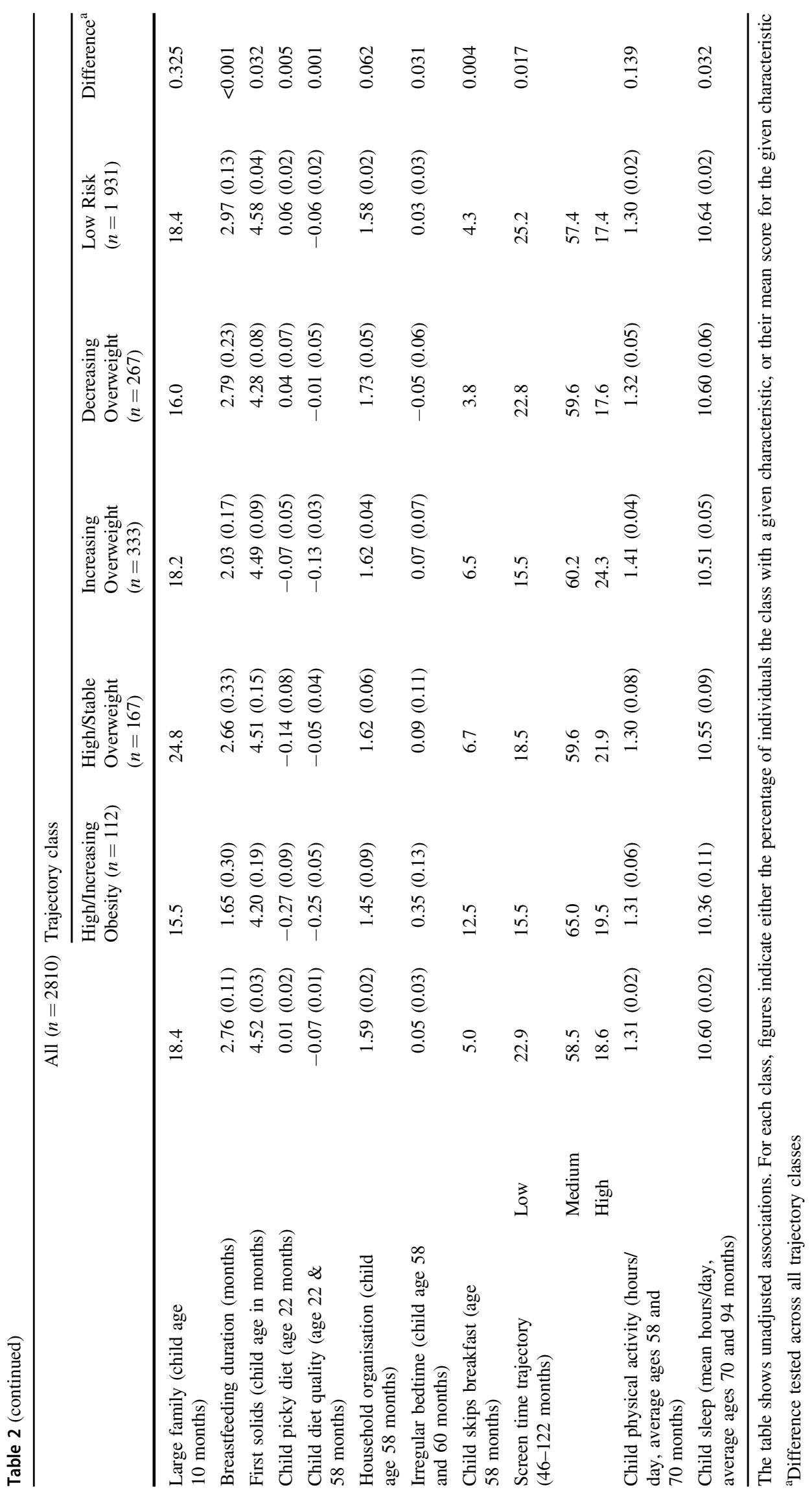




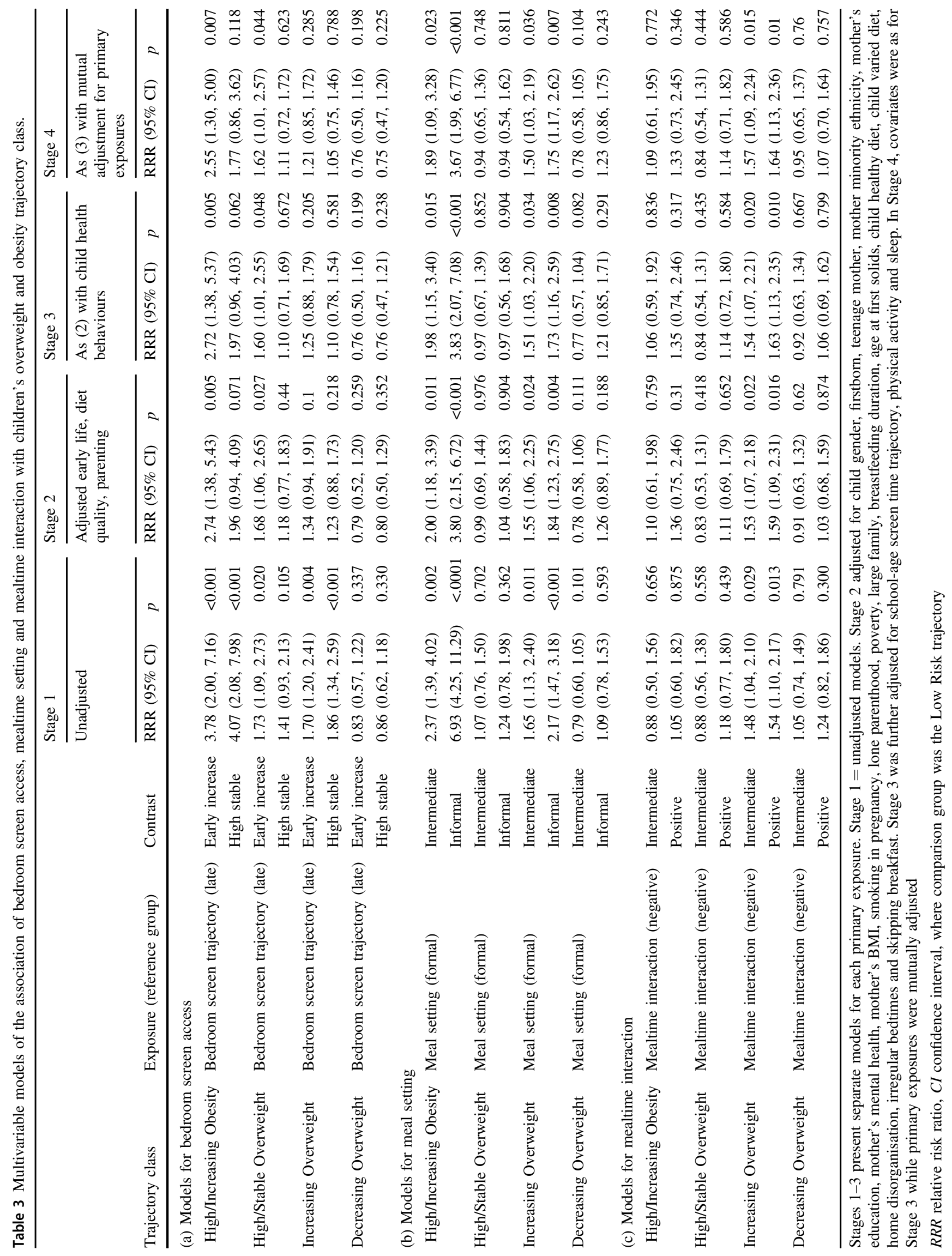


Table 3 part (c) shows estimated effects of mealtime interaction. Intermediate and positive interaction were only associated with membership of the Increasing Overweight trajectory (stage 1, unadjusted). Adjustment in stages 2 and 3 did not alter this finding.

Lastly, Table 3 stage 4 presents the effects of the three primary exposures in a mutually adjusted model, adjusting for all covariates (shown in online file S4). Interaction terms between mealtime setting and mealtime interaction were dropped, as not statistically significant (joint test $p=0.222$ ). Mutually adjusted estimates (viewing each as potential confounders for the others) were largely unchanged from those in stage 3, suggesting that the exposure effects were independent of one other.

\section{Discussion}

In this large population-based sample of Scottish children we found five different overweight/obesity trajectories across a 6-year period spanning preschool age to late middle childhood. Like others [20-24], we found a majority "healthy weight" trajectory (two-thirds of children), together with several higher-risk trajectories (known to pose later health risks [21-23]) and a decreasing trajectory [23].

The family mealtime environment and provision of bedroom screens differentiated the higher-risk trajectories from the healthy weight trajectory, even after allowing for a wide range of confounders. Children in the High/Increasing Obesity and Increasing Overweight trajectories were more likely to eat main meals in a relatively informal setting, involving mealtime screen use, sitting in a non-dining area, and not at a table. Children in the High/Increasing Obesity and High/Stable Overweight trajectories tended to have earlier access to bedroom screens. Children in the Increasing Overweight trajectory were more likely to experience positive mealtime interaction. To our knowledge, this is the first study linking aspects of the mealtime environment and timing of bedroom screen access to different patterns of children's weight gain over an extended period.

Of the three factors considered, informal mealtime setting was the only one associated with both trajectories characterised by weight gain over the study period. In contrast, mealtime setting did not differentiate children with stable or decreasing overweight patterns from healthy weight children. Estimated effects of mealtime setting were robust to adjustments for other confounders, including maternal BMI (a likely proxy for an obesogenic home environment), together with household organisation and routines, children's diet patterns and other health-related behaviours. Further research is required to assess the role of different subcomponents of mealtime setting such as screen use and sitting at a table, as we did not have sufficiently robust measures to investigate these separately. Despite this limitation, our findings tend to support other research linking mealtime screen use [10, 14] and not eating in a dining area [28] to children's obesity. Mechanisms could include increased food advertising exposure, interference with appetite regulation $[12,13]$, and lower parental monitoring and support for healthy eating, although we were unable to explore these [14, 28].

The social context of meals was also important, with the Increasing Overweight trajectory characterised by more positive mealtime interaction. This contrasts with protective effects of positive mealtime climate found by others [5, 7]. Although our measure did not specifically capture negative interactions, results appear in line with research implicating their protective role in challenging eating patterns [9]. Positive mealtime climate could also reflect instrumental feeding involving energy-dense food as a reward [51] and/ or reverse causation, if mothers felt gratified by greater food consumption. In our study the effects of mealtime social and physical context appeared independent, and we found no evidence for an interactive effect on children's pattern of weight gain.

Our findings in relation to bedroom screens extend other longitudinal research confined to two time points $[17,18]$, in demonstrating an association with higher-risk weight status over an extended period. After allowing for early life factors, household organisation and routines, and early diet patterns, the effect of bedroom screen access was only apparent for the two trajectories containing a high proportion of children already overweight or obese at the beginning of the study. This suggests that bedroom screens maintained, rather than increased, children's weight status. Like the earlier studies, we found little evidence that overall screen use, physical activity and sleep were important explanations for the effect of bedroom screens on children's overweight and obesity $[17,18]$. Findings potentially point to alternative mechanisms, such as bedroom exposure to food advertising and snacking on junk foods, although we lacked information on these. Nonetheless, the independence of bedroom device effects from mediation or confounding by overall screen use, sleep and physical activity may also reflect a degree of measurement error since children's bedroom activities may be difficult for parents to estimate accurately.

Our study has some weaknesses, including reliance on one parent (usually the mother) for information. Measures available reflect constraints dictated by a large multipurpose study. At younger ages, our measures of mealtime and bedroom screens were restricted to television; but at older ages included other less-studied screen devices, which may vary in importance for obesity risk [52]. Mealtime measures did not, unlike bedroom screen trajectory measures, capture change over time. Similarly, trajectories of overweight and 
obesity do not indicate the degree of change in BMI over time. Some inconsistencies in statistical significance of findings (such as the effects of high stable vs. early increase in bedroom screens for the High/Increasing Obesity trajectory) may reflect a lack of statistical power in relation to small trajectory classes. The Decreasing Overweight trajectory did not clearly differ from the Low Risk trajectory on any of the primary exposures. This may be due to measurement limitations discussed above, or to other factors contributing to improved weight status. Future research should explore this. Use of a representative population sample permits greater generalisability of findings, although our control for ethnicity was limited due to the predominantly White composition. Strengths include objective measures of BMI throughout childhood, and adjustment for a detailed history of background confounders. Nevertheless, estimated effects assume no unmeasured confounding, reverse causation, selection or measurement bias.

Our study extends previous research on children's weight status at a single time point $[5,7-10,17,28,34,53]$, in suggesting that modifiable aspects of bedrooms and mealtimes act in an additive manner to shape the pattern of development of overweight and obesity across several years. Mealtime setting and bedroom screen access were both important for children following the highest-risk trajectory, who all became obese by age 10 . More research is required, to extend our findings to preschool age where effects may not be as consistent [54]; and to examine mealtime interaction in more detail. Reducing bedroom screen use and adopting a more formal mealtime setting may help combat the development of obesity among schoolage children, although very few existing interventions have targeted these $[55,56]$. With near-universal integration of screens into the home environment, altering specific practices may be more readily actionable than reducing overall screen time. It seems likely, however, that families will need support to challenge existing habits. Here, interventions to improve media literacy [2]; measures reducing children's exposure to digital food advertising [57] employment policies enabling parents to "switch off" workplace contact at home [58]; and housing space standards ensuring adequate living and dining areas [59] may all have a part to play.

Acknowledgements This research was supported by Medical Research Council grants MC_UU_12017/11 and MC_UU_12017/13, and Chief Scientist Office Grants SPHSU11 and SPHSU13. Anna Pearce is additionally funded by a Wellcome Trust University Award (205412/Z/16/Z). The Growing Up in Scotland study is funded by the Scottish Government. The funder had no part in: the design and conduct of the study; collection, management, analysis, and interpretation of the data; or preparation, review, and approval of the manuscript. We thank Paul Bradshaw and the team at ScotCen Social Research for data collection, and we are grateful to the families who participated in the study.

\section{Compliance with ethical standards}

Conflict of interest The authors declare that they have no conflict of interest.

Publisher's note Springer Nature remains neutral with regard to jurisdictional claims in published maps and institutional affiliations.

\section{References}

1. Harrison K, Bost KK, McBride BA, Donovan SM, GrigsbyToussaint DS, Kim J, et al. Toward a developmental conceptualization of contributors to overweight and obesity in childhood: the six-cs model. Child Dev Perspect. 2011;5:50-8.

2. Fiese BH, Schwartz M. Reclaiming the family table: mealtimes and child health and wellbeing. Social Policy Rep. 2008;22:1-20.

3. Livingstone S. Half a century of television in the lives of our children. The annals of the American Academy of Political and Social Science. 2009;625:151-63.

4. Ofcom. Children and parents: media use and attitudes report 2018. London, Ofcom; 2019.

5. Fiese BH, Hammons A, Grigsby-Toussaint D. Family mealtimes: a contextual approach to understanding childhood obesity. Econ Hum Biol. 2012;10:365-74.

6. Baker S, Morawska A, Mitchell A. Promoting children's healthy habits through self-regulation via parenting. Clin. Child Fam Psychol. Rev. 2019;22:52-62.

7. Berge JM, Rowley S, Trofholz A, Hanson C, Rueter M, MacLehose RF, et al. Childhood obesity and interpersonal dynamics during family meals. Pediatrics. 2014;134:923-32.

8. Burnier D, Dubois L, Girard M. Arguments at mealtime and child energy intake. J Nutr Educ Behav. 2011;43:473-81.

9. Hughes SO, Power TG, Papaioannou MA, Cross MB, Nicklas TA, Hall SK, et al. Emotional climate, feeding practices, and feeding styles: an observational analysis of the dinner meal in Head Start families. Int J Behav Nutr Phys Act. 2011;8:60.

10. Ghobadi S, Hassanzadeh-Rostami Z, Salehi-Marzijarani M, Bellissimo N, Brett NR, de Zepetnek JOT, et al. Association of eating while television viewing and overweight/obesity among children and adolescents: a systematic review and meta-analysis of observational studies. Obes Rev. 2018;19:313-20.

11. Russell SJ, Croker H, Viner RM. The effect of screen advertising on children's dietary intake: a systematic review and metaanalysis. Obes Rev. 2019;20:554-68.

12. Andreyeva T, Kelly IR, Harris JL. Exposure to food advertising on television: associations with children's fast food and soft drink consumption and obesity. Econ Hum Biol. 2011;9:221-33.

13. Marsh S, Mhurchu CN, Maddson R. The non-advertising effects of screen-based sedentary activities on acute eating behaviours in children, adolescents, and young adults. A systematic review. Appetite. 2013;71:259-73.

14. Horning ML, Schow R, Friend SE, Loth K, Neumark-Sztainer D, Fulkerson JA. Family dinner frequency interacts with dinnertime context in associations with child and parent bmi outcomes. J Family Psychol. 2017;31:945-51.

15. Trofholz AC, Tate AD, Miner MH, Berge JM. Associations between TV viewing at family meals and the emotional atmosphere of the meal, meal healthfulness, child dietary intake, and child weight status. Appetite. 2017;108:361-6.

16. Wenhold $\mathrm{H}$, Harrison K. Television use and family mealtimes among a sample of US families with preschoolers. J Children Media. 2018;12:98-115.

17. Gilbert-Diamond D, Li Z, Adachi-Mejia AM, McClure AC, Sargent JD. Association of a television in the bedroom with increased 
adiposity gain in a nationally representative sample of children and adolescents. JAMA Pediatrics. 2014;168:427-34.

18. Heilmann A, Rouxel P, Fitzsimons E, Kelly Y, Watt RG. Longitudinal associations between television in the bedroom and body fatness in a UK cohort study. Int J Obes. 2017;41:1503.

19. Dube N, Khan K, Loehr S, Chu Y, Veugelers P. The use of entertainment and communication technologies before sleep could affect sleep and weight status: a population-based study among children. Intl J Behav Nutr Phys Acti. 2017;14:97.

20. Balistreri KS, Van Hook J. Trajectories of overweight among us school children: a focus on social and economic characteristics. Mater Child Health J. 2011;15:610-9.

21. Ventura AK, Loken E, Birch LL. Developmental trajectories of Girls' BMI across childhood and adolescence. Obesity. 2009;17:2067-74.

22. Ziyab AH, Karmaus W, Kurukulaaratchy RJ, Zhang HM, Arshad $\mathrm{SH}$. Developmental trajectories of body mass Index from infancy to 18 years of age: prenatal determinants and health consequences. J Epidemiol Community Health. 2014;68:934-41.

23. Kelly Y, Patalay P, Montgomery S, Sacker A. BMI development and early adolescent psychosocial well-being: UK Millennium Cohort Study. Pediatrics. 2016;138:e20160967.

24. Li C, Goran MI, Kaur H, Nollen N, Ahluwalia JS. Developmental trajectories of overweight during childhood: role of early life factors. Obesity. 2007;15:760-71.

25. ScotCen Social Research. Growing Up in Scotland: Cohort 1, Sweeps 1-8, 2005-15: Special Licence Access [data collection]. SN:5760. 14th ed. Essex: University of Essex, UK Data Service; 2019.

26. Bradshaw P, Tipping S, Marryat L, Corbett J. Growing Up in Scotland Sweep 1 - 2005 user guide. Edinburgh, Scottish Centre for Social Research; 2007.

27. Cole TJ, Bellizzi MC, Flegal KM, Dietz WH. Establishing a standard definition for child overweight and obesity worldwide: international survey. BMJ. 2000;320:1240.

28. Wansink B, van Kleef E. Dinner rituals that correlate with child and adult BMI. Obesity. 2014;22:E91-E95.

29. Jenkinson C, Layte R. Development and testing of the UK SF-12 (short form health survey). J Health Services Res Policy. 1997;2:14-8.

30. Henry JD, Crawford JR. The short-form version of the Depression Anxiety Stress Scales (DASS-21): construct validity and normative data in a large non-clinical sample. Br J Clin Psychol. 2005;44:227-39.

31. Newby PK. Are dietary intakes and eating behaviors related to childhood obesity? A comprehensive review of the evidence. J Law Med Ethics. 2007;35:35-60.

32. Antoniou EE, Roefs A, Kremers SPJ, Jansen A, Gubbels JS, Sleddens EFC, et al. Picky eating and child weight status development: alongitudinal study. J Hum Nutr Diet. 2016;29:298-307.

33. Avery A, Anderson C, McCullough F. Associations between childrenas diet quality and watching television during meal or snack consumption: a systematic review. Mater Child Nutr. 2017;13:e12428.

34. Berge JM, Jin SW, Hannan P, Neumark-Sztainer D. Structural and interpersonal characteristics of family meals: associations with adolescent body mass index and dietary patterns. J Acad Nutr Diet. 2013;113:816-22.

35. Cameron AJ, van Stralen MM, Brug J, Salmon J, Bere E, ChinApaw MJM, et al. Television in the bedroom and increased body weight: potential explanations for their relationship among European schoolchildren. Pediatr Obes. 2013;8:130-41.

36. Cole NC, Musaad SM, Lee SY, Donovan SM, Team SK. Home feeding environment and picky eating behavior in preschool-aged children: a prospective analysis. Eat Behav. 2018;30:76-82.
37. Bates CR, Buscemi J, Nicholson LM, Cory M, Jagpal A, Bohnert AM. Links between the organization of the family home environment and child obesity: a systematic review. Obes Rev. 2018;19:716-27.

38. Emond JA, Tantum LK, Gilbert-Diamond D, Kim SJ, Lansigan RK, Neelon SB. Household chaos and screen media use among preschool-aged children: a cross-sectional study. Bmc Public Health. 2018;18:1210.

39. Martin-Biggers J, Quick V, Zhang M, Jin YH, Byrd-Bredbenner C. Relationships of family conflict, cohesion, and chaos in the home environment on maternal and child food-related behaviours. Mater Child Nutr. 2018;14:e12540.

40. Matheny JAP, Wachs TD, Ludwig JL, Phillips K. Bringing order out of chaos: psychometric characteristics of the confusion, hubbub, and order scale. J Applied Dev Psychol. 1995;16:429-44.

41. Zhang G, Wu L, Zhou LL, Lu WF, Mao CT. Television watching and risk of childhood obesity: a meta-analysis. Eur J Public Health. 2016;26:13-8.

42. Birken CS, Maguire J, Mekky M, Manlhiot C, Beck CE, Jacobson $\mathrm{S}$, et al. Parental factors associated with screen time in pre-school children in primary-care practice: a TARGet Kids! study. Public Health Nutr. 2011;14:2134-8.

43. Pearson N, Biddle SJH, Griffiths P, Johnston JP, Haycraft E. Clustering and correlates of screen-time and eating behaviours among young children. BMC Public Health. 2018;18:753.

44. Jimenez-Pavon D, Kelly J, Reilly JJ. Associations between objectively measured habitual physical activity and adiposity in children and adolescents: systematic review. Int $\mathrm{J}$ Pediatr Obes. 2010;5:3-18

45. Borghese MM, Tremblay MS, Katzmarzyk PT, Tudor-Locke C, Schuna JM, Leduc G, et al. Mediating role of television time, diet patterns, physical activity and sleep duration in the association between television in the bedroom and adiposity in 10 year-old children. Int J Behav Nutr Phys Activity. 2015;12:60.

46. Fatima Y, Doi SAR, Mamun AA. Longitudinal impact of sleep on overweight and obesity in children and adolescents: a systematic review and bias-adjusted meta-analysis. Obes Rev. 2015;16:137-49.

47. Nuutinen T, Ray C, Roos E. Do computer use, TV viewing, and the presence of the media in the bedroom predict school-aged children's sleep habits in a longitudinal study? Bmc Public Health. 2013;13:684.

48. Muthén LK, Muthén BO. Mplus user's guide. 8th ed. Muthén \& Muthén: Los Angeles, CA; 1998-2017.

49. Jung T, Wickrama KAS. An introduction to latent class growth analysis and growth mixture modeling. Social Personal Psychol Compass. 2008;2:302-17.

50. StataCorp LLC. Stata/SE 14.2. College Station, TX: StataCorp LLC; 2018.

51. Rodgers RF, Paxton SJ, Massey R, Campbell KJ, Wertheim EH, Skouteris $\mathrm{H}$, et al. Maternal feeding practices predict weight gain and obesogenic eating behaviors in young children: a prospective study. Int J Behav Nutr Phys Activity. 2013;10:24.

52. Chaput J-P, Leduc G, Boyer C, Belanger P, LeBlanc AG, Borghese MM, et al. Electronic screens in children's bedrooms and adiposity, physical activity and sleep: Do the number and type of electronic devices matter? Can J Public Health. 2014;105: E273-E279.

53. Heilmann A, Rouxel P, Fitzsimons E, Kelly Y, Watt RG. Longitudinal associations between television in the bedroom and body fatness in a UK cohort study. Int J Obes. 2017;41:1503-9.

54. Collings PJ, Kelly B, West J, Wright J. Associations of TV viewing duration, meals and snacks eaten when watching $\mathrm{TV}$, and a TV in the bedroom with child adiposity. Obesity. 2018;26:1619-28. 
55. Altenburg TM, Kist-van Holthe J, Chinapaw MJM. Effectiveness of intervention strategies exclusively targeting reductions in children's sedentary time: a systematic review of the literature. Int J Behav Nutr Phys Activity. 2016;13:65.

56. Haines J, McDonald J, O'Brien A, Sherry B, Bottino CJ, Schmidt ME, et al. Healthy habits, happy homes: randomized trial to improve household routines for obesity prevention among preschool-aged children. JAMA Pediatr. 2013;167:1072-9.
57. Smith R, Kelly B, Yeatman H, Boyland E. Food marketing influences childrenas attitudes, preferences and consumption: a systematic critical review. Nutrients. 2019;11: e875.

58. King S. Should employees have a 'right to disconnect'? London: People management, CIPD; 2018.

59. HATC Limited. Housing space standards. London, Greater London Authority; 2006. 